\title{
Development of Whole Self-Capacitance Proximity and Tactile Skin Sensor for Human Collaborative Robot
}

\author{
Satoshi Tsuji ${ }^{\mathrm{a},},{ }^{*}$, Teruhiko Kohama ${ }^{\mathrm{a}}$ \\ ${ }^{a}$ Fukuoka University, 8-19-1 Nanakuma, Jonan-ku, Fukuoka 814-0180, Japan \\ *Corresponding Author: tsuji@fukuoka-u.ac.jp
}

\begin{abstract}
Human collaborative robots (HCRs) which work in a same place with peoples have become attractive in recent society. We have proposed a self-capacitance proximity and tactile skin sensor for HCRs. A structure of the sensor is simple because this sensor consists of two electrodes $\left(E_{1}\right.$ and $\mathrm{E}_{2}$ ) and an elastic body. A measurement system is also simple because only self-capacitance measurement is used to detect objects both before and after contact. In this paper, we produced ten-sensor system to cover a whole robot surface. This sensor can detect objects before contact, can detect pressures and positions after contact. In addition, we propose an operating method using the sensor for safety and workability of HCRs.
\end{abstract}

Keywords: Robot, Proximity sensor, Tactile sensor

\section{Introduction}

Most of current robots are industrial robots that work mainly in factories. Many of work spaces of these industrial robots are separated from peoples by safety fences to ensure safety. Recently, human collaborative robots (HCRs) which work with peoples on a same work space have attracted attention. A workability and space saving can be expected when HCRs work in a same space of peoples. HCRs require various sensors for workability and safety in collaboration with humans. Among them, skin sensors covering an entire robot are important for detecting contact condition with objects.

An objective of this study is to establish a simplified system of proximity and tactile skin sensor for HCRs. Fig. 1 shows a goal image of the proposed sensor system on a HCR that is work with humans. The skin sensor increases a workability and safety of HCR because the skin sensor constantly measures a state of near the robot surface, and uses to avoid contact with objects including humans, and to intuitive operating the robot.

Recent years, several types of tactile skin sensors for robot have been developed ${ }^{(1-5)}$. Using optical element to detect the contact pressure has proposed $(1,2)$. A cut-and-paste tactile sensors (1) which consist of the photo-reflector and urethane foam has network of self-contained modules consisting of tiny pressure-sensitive elements which communicate through a serial bus. A skin sensor using LED, photo transistor and silicone cap can detect three components of an applied force from contact ${ }^{(2)}$. Using capacitance measurement is proposed ${ }^{(3,4)}$. A tactile sensor using two type of urethane form and electors can detects contact force and contact area (3). A triangular modules capacitance skin sensor can detect pressure ${ }^{(4)}$. These sensors have a measurement IC in the sensor, and can be connected to other sensors to cover large area of a robot surface. A method of low wiring and high response speed has been proposed ${ }^{(5)}$. However, these tactile sensors are difficult to detect an object before a touch, and to avoid an

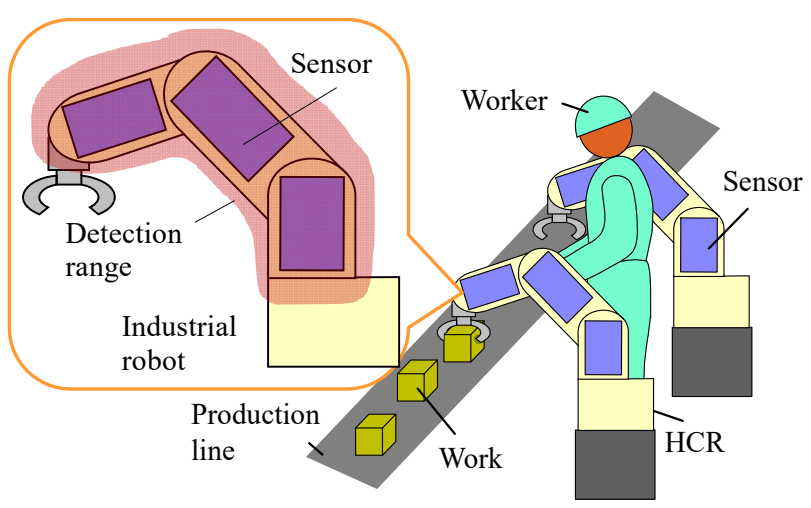

Fig. 1. Goal image of this study. 
object before contact.

HCRs need to avoid a contact with objects using proximity skin sensors. Proximity sensors for a robot have been proposed (6-8). A proximity sensor using optical element for low wiring and high response speed has been proposed ${ }^{(6)}$. The proximity sensor can detect an object and its center point before contact. A proximity sensor using capacitance measurement is proposed ${ }^{(7,8)}$. However, these proximity sensor cannot detect a contact condition.

To detect an object both before and after contact, a proximity and tactile sensor are proposed ${ }^{(9-11)}$. A robotic skin sensor using optical element can detect an object before and after contact ${ }^{(9)}$. However, it may be difficult to separate a proximity state and a contact state because proximity and contact information obtain by same information. Also, a tactile and proximity sensor using mutual capacitance measurement can detect an object at proximity range and on a contact ${ }^{(10,11)}$. However, a mutual capacitance measurement requires binary electrodes, and a structure flexibility of a sensor is thereby degraded.

We have developed a self-capacitance proximity and tactile skin sensor ${ }^{(12)}$. The self-capacitance measurement requires single electrode. The sensor structure is simple because the sensor consists of two measurement electrodes and an elastic body. The measurement system is also simple because only self-capacitance measurement is used to the measurement. The sensor on a robot can detect objects both before and after contact.

In this paper, we produced ten-sensor system to cover a whole robot surface. This sensor can detect objects before contact, can detect pressures and positions after contact. In addition, we propose an operating method using the sensor for safety and workability of HCRs. In this propose method, HCRs with sensors can be operated intuitive from sensor information without a teach pendant.

\section{Principle}

In this proposed method ${ }^{(12)}$, only self-capacitance measurement is used to detect an object both before and after contact. A self-capacitance is a capacitance between single measurement electrode and ground, and it is easy to change a size and a shape of a sensor. Fig. 2 (a) shows a schematic diagram of the proposed sensor. The sensor consist of an upper electrode $\left(E_{1}\right)$, lower electrodes $\left(E_{2}\right)$, and an elastic body. A self-capacitance between $E_{1}$ or $E_{2}$ and ground is measured by switching between $E_{1}$ and $E_{2}$. Figs. 2 (b) and (c) show an image of a proximity and (a)
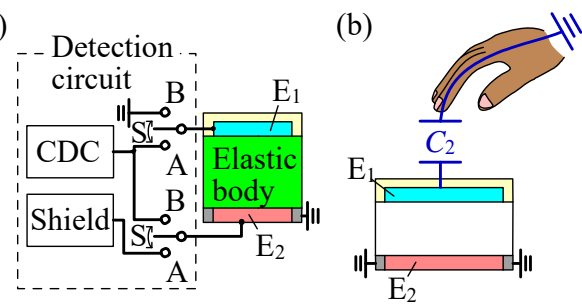

(c)

Fig. 2. Measurements by the proposed method.

contact measurement of the sensor. In Fig. 2(b), $E_{1}$ is connected to a capacitance digital converter (CDC), a self-capacitance $\left(C_{2}\right)$ of $E_{1}$ is measured (Connection A). Here, $E_{2}$ is connected to a shield. In Fig. 2(c), $E_{2}$ is connected to $\mathrm{CDC}$, and a self-capacitance $\left(C_{1}\right)$ between $\mathrm{E}_{2}$ and $E_{1}$ which is connected to ground, is measured (Connection B). In connection A, when the object approaches the sensor, $C_{2}$ changes according to both a permittivity of the object and a distance between the sensor and the object. Thus, the sensor can detect the object using $C_{2}$ before contact. Furthermore, the sensor can distinguish a material in the object using $C_{2}$ upon contact. In connection $\mathrm{B}$, when an object approaches the sensor, $C_{1}$ do not changes because $E_{1}$ connected ground. When the object touches the sensor, $C_{1}$ changes according to an indentation which is equal to a distance between $E_{2}$ and $E_{1}$. Thus, the sensor can detect the contact condition using $C_{1}$. Therefore, the sensor can detect an object before and after contact using the self-capacitance measurement ${ }^{(12)}$.

\section{Prototype Sensors}

A structure of a prototype sensor I shows in Fig. 3(a), and Fig. 3(b) is shown prototype ten-sensor. In our design, $\mathrm{E}_{1}$ is large to increase sensitivity at a proximity range, and $E_{2}$ is small to realize a high spatial resolution on contact. The size of the sensors is $150 \times 75 \mathrm{~mm}$ (Sensors I, III, IX and X), $90 \times 60 \mathrm{~mm}$ (Sensors II and IV), $60 \times 70 \mathrm{~mm}$ (Sensors V and VII), and $140 \times 80 \mathrm{~mm}$ (Sensors VI and VIII) for attachment to a robot arm (DENSO, VS-050). Here, Sensors I, III, VI, VIII, IX and X consist of $2 \times 3$ electrodes for $\mathrm{E}_{2}$ and one electrode for $\mathrm{E}_{1}$. Sensors II, IV, V and VII consist of $2 \times 2$ electrodes for $\mathrm{E}_{2}$ and one electrode for $E_{1}$. A urethane gel (thickness: $2 \mathrm{~mm}$, hardness: 0 (ASKER C), UG in Fig. 3(a)) is set between $E_{1}$ and $E_{2}$. A silicone sheet (thickness: $0.1 \mathrm{~mm}, \mathrm{Si}$ in Fig. 3(a)) is placed on the sensor surface to isolate $E_{1}$ from an object. A grounded electrode $(\mathrm{G})$ and shield electrodes $(\mathrm{S})$ are set on a back of the sensor and around $\mathrm{E}_{2}$ to reduce electrical noise 

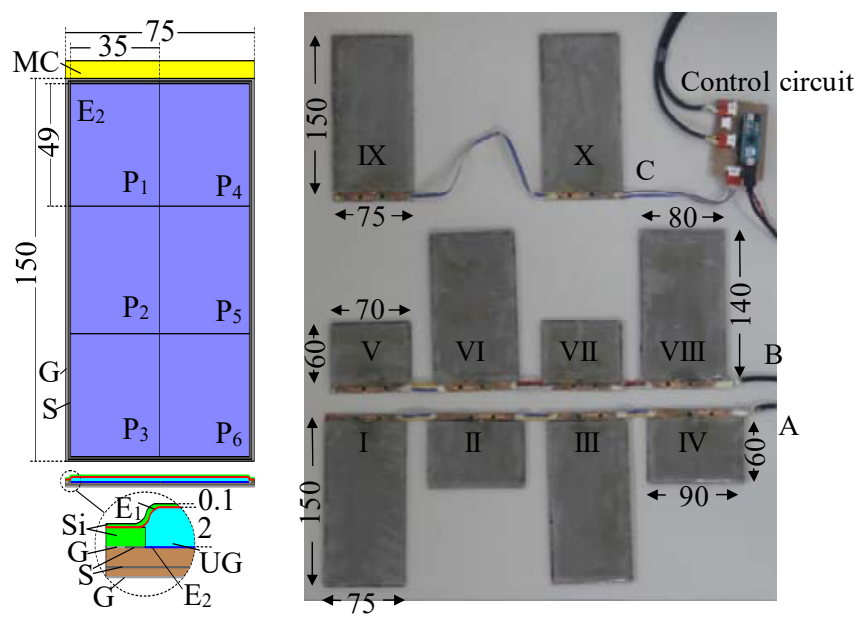

Fig. 3. Schematic diagram of the proposed sensor. $E_{1}$ and $E_{2}$ : Electrodes, UG: Urethane gel, Si: Silicone, S: Shield, G: ground, B: base, MC: Measurement circuit. (Dimensions in $\mathrm{mm})$.

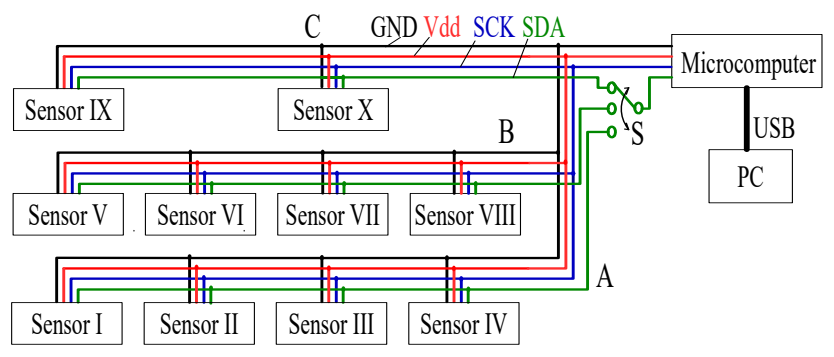

Fig. 4. Measurement system.

from a robot. Self-capacitances are measured by a measurement circuit (MC), which is attached to a side of the sensor to reduce electrical noise from wiring. MC consists of a capacitance measurement IC (Analog Devices, AD7147 is CDC), and an analog switch which changes an electrical condition of $\mathrm{E}_{1}$. Connection of $\mathrm{A}$ and $\mathrm{B}$ are continuously switched using a microcontroller and the analog switches.

Figure 4 is shown the measurement system. Measured data (16 bit) by each AD7147 (slave device) are sent to the microcomputer (master device) by $\mathrm{I}^{2} \mathrm{C}$ serial interface bus. The measured data are sent to a PC from the microcomputer by USB. $\mathrm{I}^{2} \mathrm{C}$ serial interface bus uses four lines which are serial data line (SDA), serial clock line (SCL), power line (Vdd) and ground line (GND). Four sensors can be connected with one $\mathrm{I}^{2} \mathrm{C}$ line because $\mathrm{AD} 7147$ has four $\mathrm{I}^{2} \mathrm{C}$ device addresses. The sensor system is divided into three parts $(\mathrm{A}-\mathrm{C})$. Each parts devices is connected to the master device by each SDA and common lines. Thus, this sensor system needs six wires which are three SDA, one SCL, one Vdd and one GND line. Each

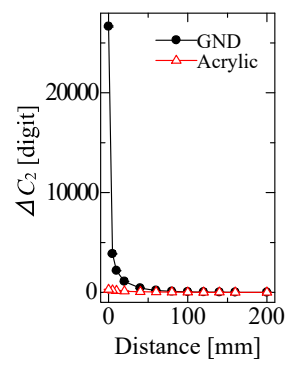

(a)

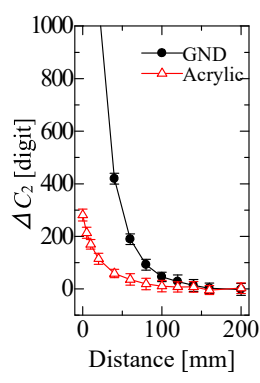

(b)

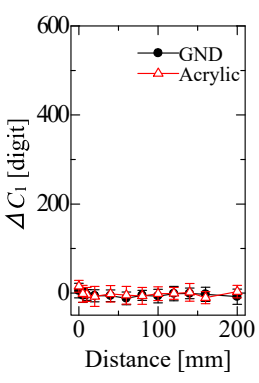

(c)
Fig. 5. Measurement results at proximity range.

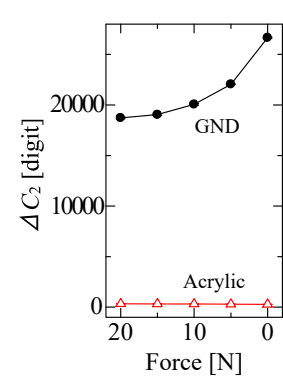

(a)

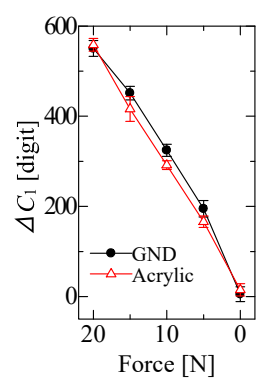

(b)

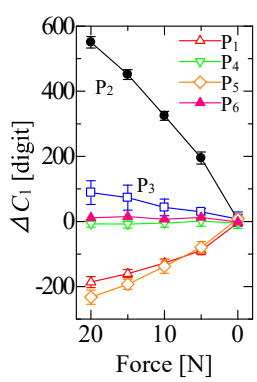

(c)
Fig. 6. Measurement results on contact.

SDA are switched by a multiplexer continuously to send measurement data. Furthermore, the sensor can be increased on same $\mathrm{I}^{2} \mathrm{C}$ line. Thus, wirings can be reduced. A unit of the measurement values indicates $\mathrm{A} / \mathrm{D}$ converted digital values (digit).

\section{Results and Discussions}

\subsection{Proximity and Contact Measurement}

The proposed sensor can detect an object both before and after contact ${ }^{(12)}$. We evaluated the proximity and contact measurements of the prototype sensors. Objects are grounded conductors (GND), which are human model, and acrylic. The size of the object is $30 \times 30 \mathrm{~mm}$. The object is set on $\mathrm{P}_{2}$ of the sensor I. The distance between the sensor and the object is changed from 0 to $200 \mathrm{~mm}$ by the robot arm. The contact condition is measured by force gauge. Fig. 5(a) shows $\Delta C_{2}$ of sensor I at proximity range, Fig. 5(b) shows the enlarged view of Fig. 5(a), and Fig. 5(c) shows $\Delta C_{1}$ of $\mathrm{P}_{2}$ on sensor I at proximity range. Here, $\Delta C_{1}$ and $\Delta C_{2}$ are the variation in $C_{1}$ and $C_{2} . \Delta C_{2}$ changes according to the distance in Figs 5(a) and (b). Thus, the sensor can detect object before contact using $\Delta C_{2}$. This sensor can detect an object (GND) within approximately $100 \mathrm{~mm}$. Here, the proximity detection distance of an object depends on an electrical characteristic of the object and an overlap between the sensor and the object ${ }^{(12)} . \Delta C_{1}$ does not change 

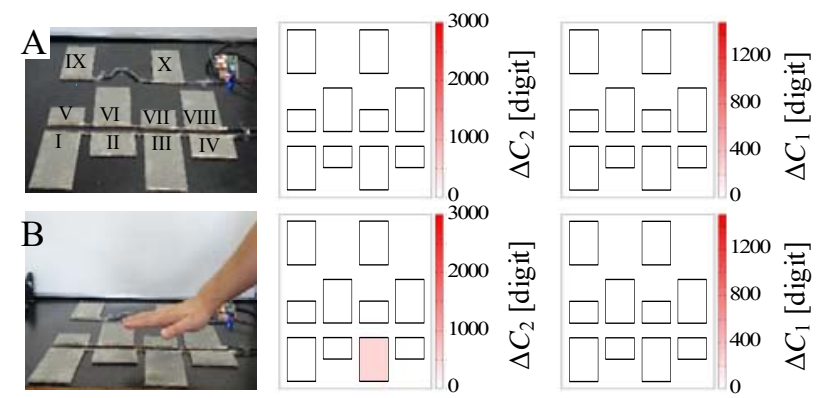

C
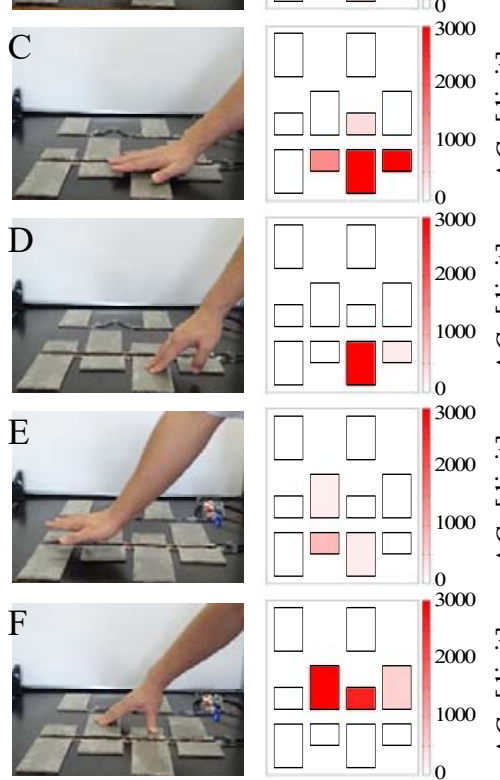

(a)

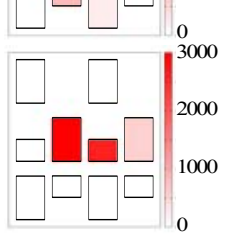

(b)
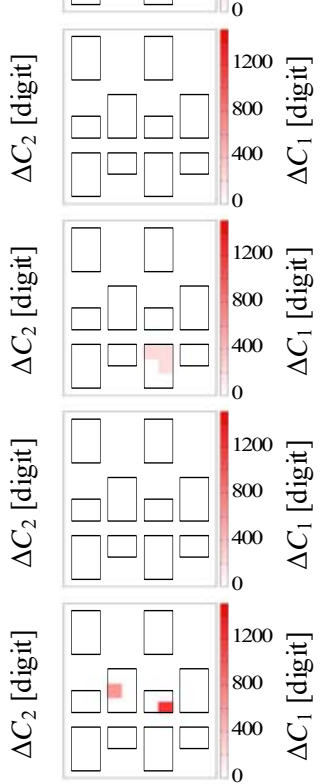

(c)

Fig. 7. Measurement results of the proposed sensors.

at non-contact, and $\Delta C_{1}$ changes after contact. Thus, the sensor can detect the contact using $\Delta C_{1}{ }^{(12)}$. Fig. 6(a) shows $\Delta C_{2}$ on contact, Fig. $6\left(\right.$ b) shows $\Delta C_{1}$ of $\mathrm{P}_{2}$ on contact, and Fig. 6(c) shows $\Delta C_{1}$ of each $E_{2}$ on contact when the object is GND. $\Delta C_{2}$ in Fig. 6(a) changes according to the permittivity of the object. Thus, the sensor can discriminate between the grounded conductor and other objects. $\Delta C_{1}$ changes according to pressure. Thus, the sensor can detect approximate pressure and its position ${ }^{(12)}$.

Figure 7 shows $\Delta C_{1}$ and $\Delta C_{2}$ of all sensors when an object is a hand. Fig. 7(a) shows a position of the hand and the sensors. The hand approached the sensor (Fig. 7 B, C and E), a finger pushed the sensor (Fig. 7 D), and two fingers pushed the sensor (Fig. $7 \mathrm{~F}$ ). Fig. 7(b) shows $\Delta C_{2}$, and Fig. 7(c) shows $\Delta C_{1} . \Delta C_{1}$ and $\Delta C_{2}$ are denoted in white in Fig. 7 when values are less than \pm 3 standard deviation (SD) lower than each value of air. In addition, $\Delta C_{2}$ in Fig. 7(b) shows 3000 digits when the values are more than 3000 digits, and $\Delta C_{1}$ in Fig. 7(c) shows 1500 digits when the values are more than 1500 digits. In Fig. 7(b), $\Delta C_{2}$ changes according to a distance at non-contact points (B, C and $\mathrm{E})$.

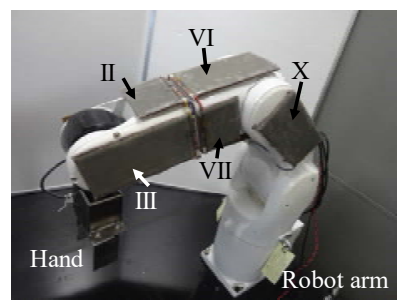

(a)

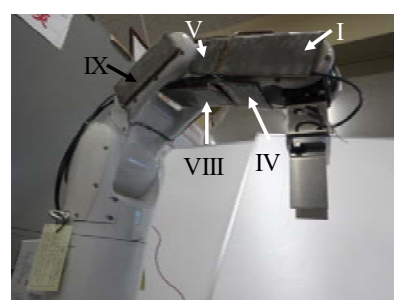

(b)
Fig. 8. Proposed sensors on the robot arm surface. (a) Top view. (b) Under view.

(a)



(b)



Fig. 9. Image of the proposed operation method.

Thus, the sensor can detect an object before contact (proximity range). In Fig. $7(\mathrm{c}), \Delta C_{1}$ changes according to a pressure and an object position when the object was pushed (D and $\mathrm{F}$ ). In addition, $\Delta C_{1}$ does not change without contact (A, B, C and E). Thus, the sensors can detect the pressure and the object position on contact. It takes approximately $61 \mathrm{~ms}$ to obtain one complete cycle of measurements (ten-sensor) with a data transmitting time from a microcomputer to PC. On the other hand, it takes approximately $35 \mathrm{~ms}$ to obtain one complete cycle of measurements (ten-sensor) without the data transmitting time from the microcomputer to PC.

\subsection{Operating Method of HCRs using the Prototype Sensor}

We propose an operating method of HCRs using the sensors on a robot. The sensors is attached on a surface of the robot arm (Fig. 8). The operation is to improve safety and workability of HCRs because peoples and robots work in a same space without safety fences. A manual operation of a robot usually use with a teach pendant. However, professional knowledge is required for a worker to operate the robot with a teach pendant. To operate a robot intuitively, we use measurement data of the sensors on the robot surface. Fig. 9 shows an image of the operating 

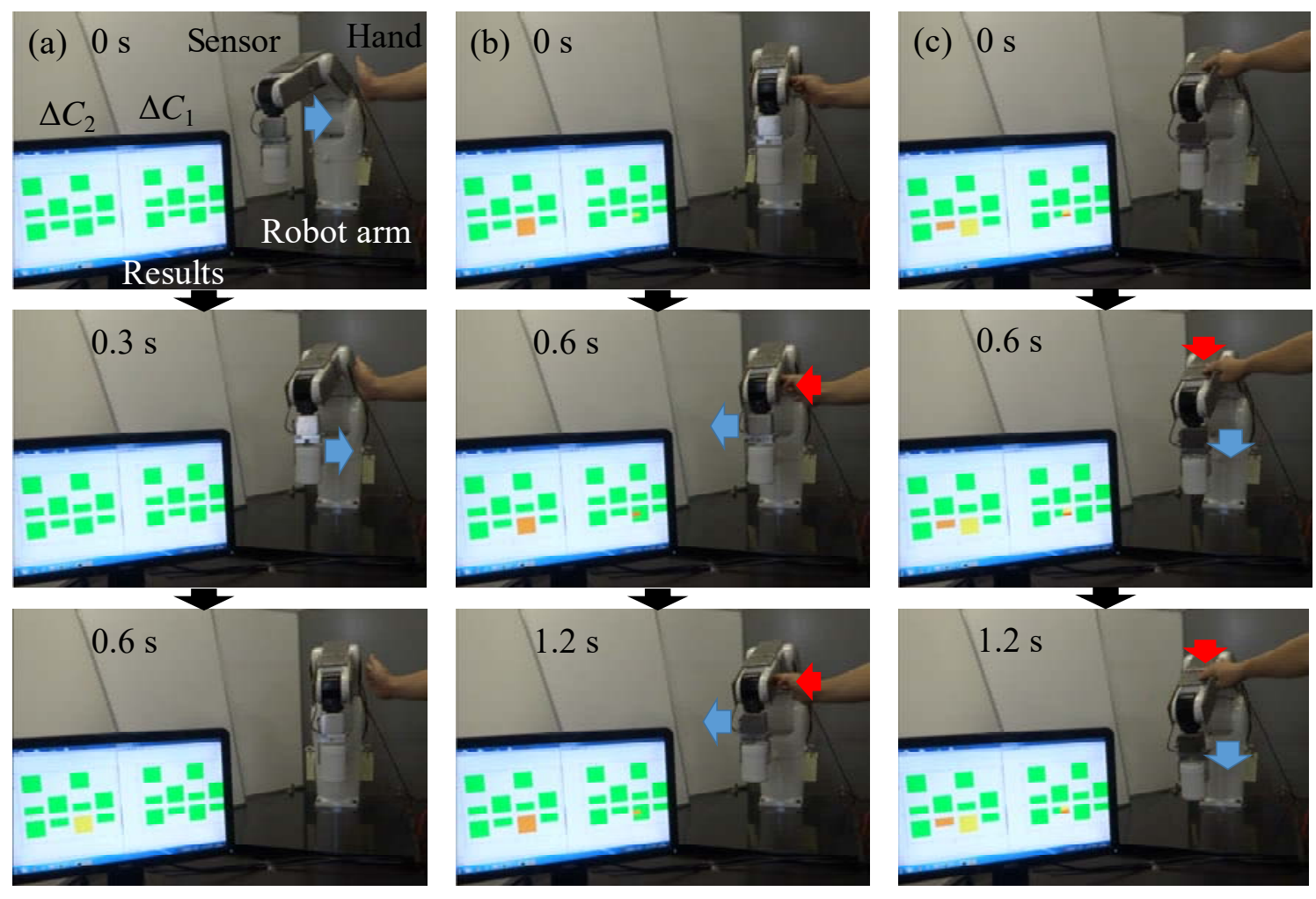

Fig. 10. Measurement and operation results of the proposed sensors on the robot arm surface.

method using the sensors. A robot speed is reduced or stopped when an object approaches the working robot (Fig. 9(a)). Thus, the sensors can improve a safety and functionality of a robot because the robot avoids unnecessary contact with objects, including humans. When a hand of a worker push one direction of the sensor, the robot moved based on a pressure and its position (Fig. 9(b)).

We evaluated the measurement of the sensors on the robot arm surface, and the operation of the robot using measurement results. In this study, we operate a robot as below.

1) When the sensor detects an object before contact, the robot is stopped until the object leaves.

2) When the sensor detects one direction pressure of an object, the robot is moved according to the pressure direction.

Fig. 10 shows measurement results and operation results. A monitor in Fig. 10 projects $\Delta C_{1}$ and $\Delta C_{2}$ of each sensors. $\Delta C_{1}$ and $\Delta C_{2}$ are denoted in green in the monitor when values are less than \pm 3 SD lower than each value of air. A red arrow shows a pressure direction by a hand. A blue arrow shows a moving direction of the robot. Fig. 10(a) shows that the robot approaches the hand, and the robot stops when $\Delta C_{2}$ of sensor III is changed, and the sensor (III) detects the object before contact. Fig. 10(b) shows that the hand push the sensor (III), the robot is moved according to a pressure direction (left side) when $\Delta C_{1}$ of sensor III is changed. Also, Fig. 10(c) shows that the hand push the sensor (II), and the robot is moved according to a pressure direction (down side) when $\Delta C_{1}$ of sensor II is changed. It was confirmed that the robot can be operated by these operations in real time. In addition, operation can be changed freely according to a setting, and operation functions can also be added. Thus, the proposed sensor can increase a safety because a robot can avoid an unexpected contact with an object including humans using sensors information. In addition, the sensor can increase functionality of a robot because the robot can be operated using the sensor without a teach pendant.

\section{Conclusions}

In this paper, we proposed the operating method of HCRs using the self-capacitance proximity and tactile skin sensor. This proposed sensor consists of two electrodes $\left(E_{1}\right.$ and $\mathrm{E}_{2}$ ) and elastic body. We produced the ten-sensor system on the same $\mathrm{I}^{2} \mathrm{C}$ bus line to covering the robot surface. The sensors on the robot surface can detect the object before and after contact. In addition, we proposed the 
following operation method.

1) When the sensor detects an object before contact, a robot is stopped until the object leaves.

2) When the sensor detects one direction pressure of an object, the robot is moved according to a pressure direction. Therefore, we think that the method is intuitive operation of HCRs without a teach pendant. The proposed sensors are thus applicable to tactile skin sensors for HCRs.

\section{Acknowledgment}

This work was supported in part by funds (No. 185009) from the Central Research Institute of Fukuoka University.

\section{References}

(1) Y. Ohmura, Y. Kuniyoshi, and A. Nagakubo : "Conformable and Scalable Tactile Sensor Skin for Curved Surfaces", in Proc. IEEE International Conference on Robotics and Automation, pp. 1348-1353, 2006

(2) A. Cirillo, F. Ficuciello, C. Natale, S. Pirozzi, and L. Villani : "A Conformable Force/Tactile Skin for Physical Human-Robot Interaction", IEEE Robotics and Automation Letters, Vol. 1, No. 1, pp. 41-48, 2016.

(3) T. Hoshi, H. Shinoda : "A Sensitive Skin Based on Touch-Area-Evaluating Tactile Elements", in Proc. IEEE Symposium on Haptic Interfaces for Virtual Environment and Teleoperator Systems, pp. 89-94, 2006

(4) P. Maiolino, M. Maggiali, G. Cannata, G. Metta, and L. Natale : "A Flexible and Robust Large Scale Capacitive Tactile System for Robots", IEEE Sensors Journal, Vol.13, No.10, pp. 3910-3917, 2013

(5) M. Shimojo, T. Araki, A. Ming, and M. Ishikawa : "A High-Speed Mesh of Tactile Sensors Fitting Arbitrary Surfaces", IEEE Sensors Journal, Vol. 10, No. 4, pp. $822-830,2010$

(6) H. Hasegawa, Y. Suzuki, A. Ming, K. Koyama, M. Ishikawa, and M. Shimojo : "Net-Structure Proximity Sensor: High-Speed and Free-Form Sensor With Analog Computing Circuit", IEEE/ASME Transactions on Mechatronics", Vol. 20, No. 6, pp. 3232-3241, 2015.

(7) T. Schlegl, T. Kroger, A. Gaschler, O. Khatib, and H. Zangl : "Virtual Whiskers-Highly Responsive Robot Collision Avoidance", in Proc. IEEE/RSJ International Conference on Intelligent Robots and Systems, pp.
5373-5379, 2013

(8) B. Sekoranja, D. Basic, M. Svaco, F. Suligoj, and B. Jerbic : "Human-Robot Interaction Based on use of Capacitive Sensors", Procedia Engineering, Vol. 69, pp. 464-468, 2014

(9) D. Hughes, J. Lammie, and N. Correll : "A Robotic Skin for Collision Avoidance and Affective Touch Recognition", IEEE Robotics and Automation Letters, Vol. 3, No. 3, pp. 1386-1393, 2018.

$(10)$ H. Lee, S. Chang, and E. Yoon : "Dual-Mode Capacitive Proximity Sensor for Robot Application: Implementation of Tactile and Proximity Sensing Capability on a Single Polymer Platform Using Shared Electrodes", IEEE Sensors Journal, Vol. 9, No. 12, pp. 1748-1755, 2009

(11)D. Goger, H. Alagi, and H. Worn : "Tactile proximity sensors for robotic applications", in Proc. Industrial Technology, pp. 978-983, 2013

(12) S. Tsuji, T. Kohama : "Proximity and Tactile Sensor Using Self-Capacitance Measurement for Human Collaboration Robots", IEEJ Transactions on Sensors and Micromachines, Vol. 138, No. 1, pp. 2-8, 2018. 